\title{
Fixed point theorems of generalized Lipschitz mappings on cone metric spaces over Banach algebras without assumption of normality
}

Shaoyuan $\mathrm{Xu}^{1 *}$ and Stojan Radenović ${ }^{2}$

"Correspondence:

xushaoyuan@126.com

'Department of Mathematics and

Statistics, Hanshan Normal

University, Chaozhou, 521041, China

Full list of author information is

available at the end of the article

\section{Springer}

\begin{abstract}
In this paper, we first present some elementary results concerning cone metric spaces over Banach algebras. Next, by using these results and the related ones about $c$-sequence on cone metric spaces we obtain some new fixed point theorems for the generalized Lipschitz mappings on cone metric spaces over Banach algebras without the assumption of normality. As a consequence, our main results improve and generalize the corresponding results in the recent paper by Liu and Xu (Fixed Point Theory Appl. 2013:320, 2013).
\end{abstract}

MSC: $54 \mathrm{H} 25 ; 47 \mathrm{H} 10$

Keywords: cone metric spaces over Banach algebras; non-normal cones; fixed point theorems; generalized Lipschitz mappings; $c$-sequences; spectral radius

\section{Introduction}

In 2007, cone metric spaces were reviewed by Huang and Zhang, as a generalization of metric spaces (see [1]). The distance $d(x, y)$ of two elements $x$ and $y$ in a cone metric space $X$ is defined to be a vector in an ordered Banach space $E$, quite different from that which is defined to be a non-negative real number in general metric spaces. They gave the version of the Banach contraction principle and other basic theorems in the setting of cone metric spaces. Later on, by omitting the assumption of normality in the results of [1], Rezapour and Hamlbarani [2] obtained some fixed point theorems, as the generalizations of the relevant results in [1]. Besides, they presented a number of examples to prove the existence of non-normal cones, which shows that such generalizations are meaningful. Since then, many authors have been interested in the study of fixed point results in the setting of cone metric spaces (see [2-10]).

Recall that a mapping $T: X \rightarrow X$ is said to be contractive if there is a constant $k \in[0,1)$ such that

$$
d(T x, T y) \preceq k d(x, y), \quad x, y \in X .
$$

The right-hand side of the inequality (1) is the vector as the result of the operation of scalar multiplication in cone metric spaces. In [1], the authors proved that there exists a unique fixed point for contractive mappings in complete cone metric spaces.

Recently, some authors investigated the problem of whether cone metric spaces are equivalent to metric spaces in terms of the existence of the fixed points of the mappings

(O2014 Xu and Radenović; licensee Springer. This is an Open Access article distributed under the terms of the Creative Commons Attribution License (http://creativecommons.org/licenses/by/2.0), which permits unrestricted use, distribution, and reproduction in any medium, provided the original work is properly cited. 
involved. They established the equivalence between some fixed point results in metric and in (topological vector space-valued) cone metric spaces see [11-14]. Actually, they showed that any cone metric space $(X, d)$ is equivalent to a usual metric space $\left(X, d^{*}\right)$, where the real-valued metric function $d^{*}$ is defined by a nonlinear scalarization function $\xi_{e}$ (see [12]) or by a Minkowski functional $q_{e}$ (see [13]). After that, some other interesting generalizations were developed. See, for instance, [4].

Very recently, Liu and $\mathrm{Xu}[15]$ introduced the concept of cone metric spaces over Banach algebras (which were called cone metric spaces over Banach algebras in [15]), replacing Banach spaces by Banach algebras as the underlying spaces of cone metric spaces. They replaced the Banach space $E$ by a Banach algebra $\mathcal{A}$ and introduced the concept of cone metric spaces over Banach algebras. In this way, they proved some fixed point theorems of generalized Lipschitz mappings with weaker and natural conditions on generalized Lipschitz constant $k$ by means of spectral radius. Note that it is significant to introduce the concept of cone metric spaces with Banach algebras since one can prove that cone metric spaces with Banach algebras are not equivalent to metric spaces in terms of the existence of the fixed points of the generalized Lipschitz mappings. As a matter of fact, Liu and Xu showed that the main results obtained in [15] could not be reduced to a consequence of corresponding results in metric spaces by means of the methods in the literature. This does bring about prosperity in the study of cone metric spaces. However, the proofs of the main results in [15] depends strongly on the condition that the underlying solid cone is normal. In this paper, we delete the superfluous assumption of normality of the paper [15] and also obtain the existence and uniqueness of the fixed point for the generalized Lipschitz mappings in the setting of cone metric spaces over Banach algebras. The methods and techniques used in this paper are quite different from those appearing in [2]. Furthermore, we give an example to support that it is meaningful to set up fixed point theorems of generalized Lipschitz mappings without the assumption of normality of the underlying solid cones.

\section{Preliminaries}

Let $\mathcal{A}$ always be a real Banach algebra. That is, $\mathcal{A}$ is a real Banach space in which an operation of multiplication is defined, subject to the following properties (for all $x, y, z \in \mathcal{A}$, $\alpha \in \mathbb{R})$ :

1. $(x y) z=x(y z)$

2. $x(y+z)=x y+x z$ and $(x+y) z=x z+y z$;

3. $\alpha(x y)=(\alpha x) y=x(\alpha y)$;

4. $\|x y\| \leq\|x\|\|y\|$.

Throughout this paper, we shall assume that a Banach algebra has a unit (i.e., a multiplicative identity) $e$ such that $e x=x e=x$ for all $x \in \mathcal{A}$. An element $x \in \mathcal{A}$ is said to be invertible if there is an inverse element $y \in \mathcal{A}$ such that $x y=y x=e$. The inverse of $x$ is denoted by $x^{-1}$. For more details, we refer to [16].

The following proposition is well known (see [16]).

Proposition 2.1 Let $\mathcal{A}$ be a Banach algebra with a unit e, and $x \in \mathcal{A}$. If the spectral radius $r(x)$ of $x$ is less than 1, i.e.,

$$
r(x)=\lim _{n \rightarrow \infty}\left\|x^{n}\right\|^{\frac{1}{n}}=\inf _{n \geq 1}\left\|x^{n}\right\|^{\frac{1}{n}}<1,
$$


then $e-x$ is invertible. Actually,

$$
(e-x)^{-1}=\sum_{i=0}^{\infty} x^{i}
$$

Remark 2.1 From [16] we see that the spectral radius $r(x)$ of $x$ satisfies

$$
r(x) \leq\|x\|
$$

for all $x \in \mathcal{A}$, where $\mathcal{A}$ is a Banach algebra with a unit $e$.

Remark 2.2 In Proposition 2.1, if the condition ' $r(x)<1$ ' is replaced by $\|x\| \leq 1$, then the conclusion remains true.

Now let us recall the concepts of cone and semi-order for a Banach algebra $\mathcal{A}$. A subset $P$ of $\mathcal{A}$ is called a cone of $\mathcal{A}$ if

1. $P$ is non-empty closed and $\{\theta, e\} \subset P$;

2. $\alpha P+\beta P \subset P$ for all non-negative real numbers $\alpha, \beta$;

3. $P^{2}=P P \subset P$

4. $P \cap(-P)=\{\theta\}$,

where $\theta$ denotes the null of the Banach algebra $\mathcal{A}$. For a given cone $P \subset \mathcal{A}$, we can define a partial ordering $\preceq$ with respect to $P$ by $x \preceq y$ if and only if $y-x \in P$. $x \prec y$ will stand for $x \preceq y$ and $x \neq y$, while $x \ll y$ will stand for $y-x \in \operatorname{int} P$, where int $P$ denotes the interior of $P$. If int $P \neq \emptyset$ then $P$ is called a solid cone.

The cone $P$ is called normal if there is a number $M>0$ such that, for all $x, y \in \mathcal{A}$,

$$
\theta \preceq x \preceq y \quad \Rightarrow \quad\|x\| \leq M\|y\| .
$$

The least positive number satisfying above is called the normal constant of $P$ [1].

In the following we always assume that $P$ is a cone in $\mathcal{A}$ with int $P \neq \emptyset$ and $\preceq$ is the partial ordering with respect to $P$.

Definition 2.1 (See $[1,15]$ and [17]) Let $X$ be a non-empty set. Suppose that the mapping $d: X \times X \rightarrow \mathcal{A}$ satisfies

1. $0 \preceq d(x, y)$ for all $x, y \in X$ and $d(x, y)=0$ if and only if $x=y$;

2. $\quad d(x, y)=d(y, x)$ for all $x, y \in X$;

3. $\quad d(x, y) \preceq d(x, z)+d(z, x)$ for all $x, y, z \in X$.

Then $d$ is called a cone metric on $X$, and $(X, d)$ is called a cone metric space over a Banach algebra $\mathcal{A}$.

For convenance, we give an example of cone metric space over Banach algebra as follows. For more examples, see [15].

Example 2.1 (See [15]) Let $\mathcal{A}=M_{n}(\mathbb{R})=\left\{a=\left(a_{i j}\right)_{n \times n} \mid a_{i j} \in \mathbb{R}\right.$, for all $\left.1 \leq i, j \leq n\right\}$ be the algebra of all $n$-square real matrices, and define the norm

$$
\|a\|=\sum_{1 \leq i, j \leq n}\left|a_{i j}\right| .
$$

Then $\mathcal{A}$ is a real Banach algebra with the unit $e$ the identity matrix. 
Let $P=\left\{a \in \mathcal{A} \mid a_{i j} \geq 0\right.$, for all $\left.1 \leq i, j \leq n\right\}$. Then $P \subset \mathcal{A}$ is a normal cone with normal constant $M=1$.

Let $X=M_{n}(\mathbb{R})$, and define the metric $d: X \times X \rightarrow \mathcal{A}$ by

$$
d(x, y)=d\left(\left(x_{i j}\right)_{n \times n},\left(y_{i j}\right)_{n \times n}\right)=\left(\left|x_{i j}-y_{i j}\right|\right)_{n \times n} \in \mathcal{A} .
$$

Then $(X, d)$ is a cone metric space over Banach algebra $\mathcal{A}$ with normality.

Example 2.2 Let $\mathcal{A}=C_{\mathbb{R}}^{1}[0,1]$ and define a norm on $\mathcal{A}$ by $\|x\|=\|x\|_{\infty}+\left\|x^{\prime}\right\|_{\infty}$ for $x \in \mathcal{A}$. Define multiplication in $\mathcal{A}$ as just pointwise multiplication. Then $\mathcal{A}$ is a real unital Banach algebra with unit $e=1$. The set $P=\{x \in \mathcal{A}: x \geq 0\}$ is a cone in $\mathcal{A}$. Moreover, $P$ is not normal (see [2]).

Let $X=\{1,2,3\}$. Define $d: X \times X \rightarrow \mathcal{A}$ by $d(1,2)(t)=d(2,1)(t)=d(2,3)(t)=d(3,2)(t)=$ $e^{t}, d(1,3)(t)=d(3,1)(t)=2 e^{t}, d(x, x)(t)=0$. We see that $(X, d)$ is a cone metric space over Banach algebra $\mathcal{A}$ without normality.

Definition 2.2 (See $[1,15]$ or [17]) Let $(X, d)$ be a cone metric space over a Banach algebra $\mathcal{A}, x \in X$ and let $\left\{x_{n}\right\}$ be a sequence in $X$. Then:

1. $\left\{x_{n}\right\}$ converges to $x$ whenever for each $c \in \mathcal{A}$ with $\theta \ll c$ there is a natural number $N$ such that $d\left(x_{n}, x\right) \ll c$ for all $n \geq N$. We denote this by $\lim _{n \rightarrow \infty} x_{n}=x$ or $x_{n} \rightarrow x$.

2. $\left\{x_{n}\right\}$ is a Cauchy sequence whenever for each $c \in \mathcal{A}$ with $\theta \ll c$ there is a natural number $N$ such that $d\left(x_{n}, x_{m}\right) \ll c$ for all $n, m \geq N$.

3. $(X, d)$ is a complete cone metric space if every Cauchy sequence is convergent.

Now, we shall appeal to the following lemmas in the sequel.

Lemma 2.1 (See [18]) If $E$ is a real Banach space with a cone $P$ and if $a \preceq \lambda a$ with $a \in P$ and $0 \leq \lambda<1$, then $a=\theta$.

Lemma 2.2 (See [9]) If $E$ is a real Banach space with a solid cone $P$ and if $\theta \preceq u \ll c$ for each $\theta \ll c$, then $u=\theta$.

Lemma 2.3 (See [9]) If $E$ is a real Banach space with a solid cone $P$ and if $\left\|x_{n}\right\| \rightarrow 0$ $(n \rightarrow \infty)$, then for any $\theta \ll c$, there exists $N \in \mathbb{N}$ such that, for any $n>N$, we have $x_{n} \ll c$.

Finally, let us recall the concept of generalized Lipschitz mapping defining on the cone metric spaces over Banach algebras, which is introduced in [15].

Definition 2.3 (See [15]) Let $(X, d)$ be a cone metric space over a Banach algebra $\mathcal{A}$. A mapping $T: X \rightarrow X$ is called a generalized Lipschitz mapping if there exists a vector $k \in P$ with $r(k)<1$ and for all $x, y \in X$, one has

$$
d(T x, T y) \preceq k d(x, y) .
$$

Remark 2.3 In Definition 2.3, we only suppose the spectral radius of $k$ is less than 1 , while $\|k\|<1$ is not assumed. Generally speaking, it is meaningful since by Remark 2.1, the condition $r(k)<1$ is weaker than that $\|k\|<1$.

Remark 2.4 If $r(k)<1$ then $\left\|k^{n}\right\| \rightarrow 0(n \rightarrow \infty)$. 


\section{Main results}

In this section, by omitting the assumption of normality of the main results in Liu and $\mathrm{Xu}$ [15], we shall prove some fixed point theorems of generalized Lipschitz mappings in the setting of cone metric spaces over Banach algebras.

We begin this section with reviewing some facts on $c$-sequence theory.

Definition 3.1 (See $[19,20])$ Let $P$ be a solid cone in a Banach space $\mathcal{A}$. A sequence $\left\{u_{n}\right\} \subset$ $P$ is a $c$-sequence if for each $c \gg \theta$ there exists $n_{0} \in \mathbb{N}$ such that $u_{n} \ll c$ for $n \geq n_{0}$.

It is easy to show the following proposition.

Proposition 3.1 (See [19]) Let $P$ be a solid cone in a Banach space $\mathcal{A}$ and let $\left\{x_{n}\right\}$ and $\left\{y_{n}\right\}$ be sequences in $P$. If $\left\{x_{n}\right\}$ and $\left\{y_{n}\right\}$ are c-sequences and $\alpha, \beta>0$, then $\left\{\alpha x_{n}+\beta y_{n}\right\}$ is a c-sequence.

In addition to Proposition 3.1 above, the following propositions are crucial to the proof of our main result.

Proposition 3.2 (See [19]) Let $P$ be a solid cone in a Banach algebra $\mathcal{A}$ and let $\left\{x_{n}\right\}$ be a sequence in $P$. Then the following conditions are equivalent:

(1) $\left\{x_{n}\right\}$ is a c-sequence.

(2) For each $c \gg \theta$ there exists $n_{0} \in \mathbb{N}$ such that $x_{n} \prec c$ for $n \geq n_{0}$.

(3) For each $c \gg \theta$ there exists $n_{1} \in \mathbb{N}$ such that $x_{n} \preceq c$ for $n \geq n_{1}$.

Proposition 3.3 Let $P$ be a solid cone in a Banach algebra $\mathcal{A}$ and let $\left\{u_{n}\right\}$ be a sequence in $P$. Suppose that $k \in P$ is an arbitrarily given vector and $\left\{u_{n}\right\}$ is a $c$-sequence in $P$. Then $\left\{k u_{n}\right\}$ is a c-sequence.

Proof Fix $c \gg \theta$. Then also $\frac{c}{m} \gg \theta$ for all $m \in \mathbb{N}$. Therefore, since $\left\{x_{n}\right\}$ is a $c$-sequence, it follows that, for each $m \in \mathbb{N}$, there exists $n_{m} \in \mathbb{N}$ such that $u_{n} \ll \frac{c}{m}$ for all $n \geq n_{m}$. Hence, for all $n \geq n_{m}$ we have $k u_{n} \preceq \frac{k c}{m}$. Now, since $\frac{k c}{m} \rightarrow \theta$ as $m \rightarrow \infty$, there exists $m_{1} \in \mathbb{N}$ such that $\frac{k c}{m} \ll c$ for all $m>m_{1}$. Hence, there exists $n_{0} \in \mathbb{N}$ such that, for all $n>n_{0}$ we have $k u_{n} \ll c$, that is, $\left\{k u_{n}\right\}$ is a $c$-sequence.

Proposition 3.4 Let $\mathcal{A}$ be a Banach algebra with a unit e, $P$ be a cone in $\mathcal{A}$ and $\preceq$ be the semi-order generated by the cone $P$. The following assertions hold true:

(i) For any $x, y \in \mathcal{A}, a \in P$ with $x \preceq y$, we have $a x \preceq a y$.

(ii) For any sequences $\left\{x_{n}\right\},\left\{y_{n}\right\} \subset \mathcal{A}$ with $x_{n} \rightarrow x(n \rightarrow \infty)$ and $y_{n} \rightarrow y(n \rightarrow \infty)$ where $x, y \in \mathcal{A}$, we have $x_{n} y_{n} \rightarrow x y(n \rightarrow \infty)$.

Proposition 3.5 Let $\mathcal{A}$ be a Banach algebra with a unit e, $P$ be a cone in $\mathcal{A}$ and $\preceq$ be the semi-order generated by the cone $P$. Let $\lambda \in P$. If the spectral radius $r(\lambda)$ of $\lambda$ is less than 1 , then the following assertions hold true:

(i) Suppose that $x$ is invertible and that $x^{-1} \succ \theta$ implies $x>\theta$, then for any integer $n \geq 1$, we have $\lambda^{n} \preceq \lambda \preceq e$.

(ii) For any $u>\theta$, we have $u \npreceq \lambda u$, i.e., $\lambda u-u \notin P$.

(iii) If $\lambda \succeq \theta$, then we have $(e-\lambda)^{-1} \succeq \theta$. 
Proof (i) Since $r(\lambda)<1$, by Proposition 2.1, we see the element $e-\lambda$ is invertible. Considering

$$
e=(e-\lambda)(e-\lambda)^{-1}=(e-\lambda) \sum_{i=0}^{\infty} \lambda^{i}
$$

we have

$$
\lambda=(e-\lambda) \sum_{i=1}^{\infty} \lambda^{i} \preceq(e-\lambda) \sum_{i=0}^{\infty} \lambda^{i}=(e-\lambda)(e-\lambda)^{-1}=e,
$$

which implies that

$$
\lambda^{n} \preceq \lambda
$$

for all $n \geq 1$ by induction. Therefore, the conclusion of (i) is true.

(ii) If it is not true, then there exists an element $u_{0} \in \mathcal{A}$ with $u_{0}>\theta$ such that

$$
u_{0} \preceq \lambda u_{0} .
$$

Hence it follows that

$$
(e-\lambda) u_{0} \preceq \theta \text {. }
$$

Then multiplying both sides with $(e-\lambda)^{-1}$, it follows that $u_{0}=\theta$, a contradiction.

(iii) It is obvious.

Remark 3.1 Proposition 3.5(ii) is the version of Lemma 2.1 in the setting of cone metric spaces over Banach algebras.

It is easy to show the following proposition by Definitions 2.1 and 3.1, so we omit its proof.

Proposition 3.6 Let $(X, d)$ be a complete cone metric space over a Banach algebra $\mathcal{A}$ and let $P$ be the underlying solid cone in Banach algebra $\mathcal{A}$. Let $\left\{x_{n}\right\}$ be a sequence in X. If $\left\{x_{n}\right\}$ converges to $x \in X$, then we have:

(i) $\left\{d\left(x_{n}, x\right)\right\}$ is a c-sequence.

(ii) For any $p \in \mathbb{N},\left\{d\left(x_{n}, x_{n+p}\right)\right\}$ is a c-sequence.

Now, we begin to present the version of the famous Banach contraction principle for generalized Lipschitz mappings in the setting of cone metric space over Banach algebra without the assumption of normality of the underlying solid cone.

Theorem 3.1 Let $(X, d)$ be a complete cone metric space over a Banach algebra $\mathcal{A}$ and let $P$ be the underlying solid cone with $k \in P$ where $r(k)<1$. Suppose the mapping $T: X \rightarrow X$ satisfies generalized Lipschitz condition:

$$
d(T x, T y) \preceq k d(x, y) \quad \text { for all } x, y \in X .
$$


Then $T$ has a unique fixed point in $X$. For any $x \in X$, iterative sequence $\left\{T^{n} x\right\}$ converges to the fixed point.

Proof Let $x \in X$ be arbitrarily given and $\operatorname{set} x_{n}=T^{n} x, n \geq 1$. We have

$$
d\left(x_{n+1}, x_{n}\right) \preceq k d\left(x_{n}, x_{n-1}\right) \preceq \cdots \preceq k^{n} d\left(x_{1}, x_{0}\right) .
$$

Thus, for $n<m$, we have

$$
\begin{aligned}
d\left(x_{n}, x_{m}\right) & \preceq d\left(x_{n}, x_{n+1}\right)+\cdots+d\left(x_{m-1}, x_{m}\right) \\
& \preceq\left(k^{n}+\cdots+k^{m-1}\right) d\left(x_{1}, x_{0}\right) \\
& =\left(e+k+\cdots+k^{m-n-1}\right) k^{n} d\left(x_{1}, x_{0}\right) \\
& \preceq\left(\sum_{i=0}^{\infty} k^{i}\right) k^{n} d\left(x_{1}, x_{0}\right) \\
& =(e-k)^{-1} k^{n} d\left(x_{1}, x_{0}\right) .
\end{aligned}
$$

By Lemma 2.3 and the fact that $\left\|k^{n}(e-k)^{-1} d\left(x_{0}, x_{1}\right)\right\| \rightarrow 0(n \rightarrow \infty)$ (noting that by Remark 2.4, $\left.\left\|k^{n}\right\| \rightarrow 0(n \rightarrow \infty)\right)$, it follows that, for any $c \in \mathcal{A}$ with $\theta \ll c$, there exists $N \in \mathbb{N}$ such that, for any $m>n>N$, we have

$$
d\left(x_{m}, x_{n}\right) \preceq k^{n}(e-k)^{-1} d\left(x_{0}, x_{1}\right) \ll c,
$$

which implies that $\left\{x_{n}\right\}$ is a Cauchy sequence.

By the completeness of $X$, there exists $x^{*} \in X$ such that $x_{n} \rightarrow x^{*}(n \rightarrow \infty)$. Furthermore, one has

$$
\begin{aligned}
d\left(T x^{*}, x^{*}\right) & \preceq d\left(T x^{*}, T x_{n}\right)+d\left(T x_{n}, x^{*}\right) \\
& \preceq k d\left(x^{*}, x_{n}\right)+d\left(x_{n+1}, x^{*}\right) .
\end{aligned}
$$

Therefore, it follows from Definition 3.1, Propositions 3.1, 3.2, 3.3, and 3.6 that we have $d\left(x^{*}, T x^{*}\right) \preceq y_{n}$ where $y_{n}$ is a $c$-sequence in cone $P$. Hence, for each $c \gg \theta$ we have $d\left(x^{*}, T x^{*}\right) \ll c$, so $d\left(x^{*}, T x^{*}\right)=\theta$ by Lemma 2.2. Thus $x^{*}$ is a fixed point of $T$.

Finally, we prove the uniqueness of the fixed point. The proof is as same as that in Theorem 2.1 in [15] since it actually does not require the assumption of normality of the underlying solid cone.

In the following, we will present some other fixed point theorems of generalized Lipschitz mappings in the setting of cone metric space over Banach algebra without the assumption of normality of the underlying solid cone. For convenience, let us give some basic results concerning spectral radius.

Lemma 3.1 Let $\mathcal{A}$ be a Banach algebra and let $x, y$ be vectors in $\mathcal{A}$. If $x$ and $y$ commute, then the following hold:

(i) $r(x y) \leq r(x) r(y)$;

(ii) $r(x+y) \leq r(x)+r(y)$;

(iii) $|r(x)-r(y)| \leq r(x-y)$. 
Lemma 3.2 Let $\mathcal{A}$ be a Banach algebra and let $\left\{x_{n}\right\}$ be a sequence in $\mathcal{A}$. Suppose that $\left\{x_{n}\right\}$ converges to $x$ in $\mathcal{A}$ and that $x_{n}$ and $x$ commute for all $n$, then we have $r\left(x_{n}\right) \rightarrow r(x)$ as $n \rightarrow \infty$.

Proof It follows from Lemma 3.1 and Remark 2.1 that

$$
\left|r\left(x_{n}\right)-r(x)\right| \leq r\left(x_{n}-x\right) \leq\left\|x_{n}-x\right\| \rightarrow 0 \quad(n \rightarrow \infty),
$$

which completes the proof.

Lemma 3.3 Let $\mathcal{A}$ be a Banach algebra and let $k$ be a vector in $\mathcal{A}$. If $0 \leq r(k)<1$, then we have $r\left((e-k)^{-1}\right) \leq(1-r(k))^{-1}$.

Proof Since $0 \leq r(k)<1$, it follows that

$$
(e-k)^{-1}=e+\sum_{i=1}^{\infty} k^{i}=e+\lim _{n \rightarrow \infty} \sum_{i=1}^{n} k^{i}
$$

Hence, it follows from Lemmas 3.1 and 3.2 that

$$
\begin{aligned}
r\left((e-k)^{-1}\right) & \leq r(e)+r\left(\lim _{n \rightarrow \infty} \sum_{i=1}^{n} k^{i}\right) \\
& \leq r(e)+\lim _{n \rightarrow \infty} \sum_{i=1}^{n}(r(k))^{i} \\
& \leq 1+\sum_{i=1}^{\infty}(r(k))^{i} \\
& =(1-r(k))^{-1},
\end{aligned}
$$

which completes the proof.

Theorem 3.2 Let $(X, d)$ be a complete cone metric space over a Banach algebra $\mathcal{A}$ and let $P$ be the underlying solid cone with $k \in P$ where $r(k)<\frac{1}{2}$. Suppose the mapping $T: X \rightarrow X$ satisfies the generalized Lipschitz condition

$$
d(T x, T y) \preceq k(d(T x, y)+d(T y, x)) \quad \text { for all } x, y \in X .
$$

Then $T$ has a unique fixed point in $X$. And for any $x \in X$, iterative sequence $\left\{T^{n} x\right\}$ converges to the fixed point.

Proof Let $x \in X$ be arbitrarily given and $\operatorname{set} x_{n}=T^{n} x, n \geq 1$. We have

$$
\begin{aligned}
d\left(x_{n+1}, x_{n}\right) & =d\left(T x_{n}, T x_{n-1}\right) \\
& \preceq k\left(d\left(T x_{n}, x_{n-1}\right)+d\left(T x_{n-1}, x_{n}\right)\right) \\
& \preceq k\left(d\left(x_{n+1}, x_{n}\right)+d\left(x_{n}, x_{n-1}\right)\right) .
\end{aligned}
$$


That is,

$$
d\left(x_{n+1}, x_{n}\right) \preceq(e-k)^{-1} k d\left(x_{n}, x_{n-1}\right) .
$$

We now prove that

$$
r\left((e-k)^{-1} k\right)<1
$$

In fact, noting that $(e-k)^{-1}$ and $k$ commute, by Lemma 3.3 above and the fact that $r(k)<\frac{1}{2}$, we have

$$
\begin{aligned}
r\left((e-k)^{-1} k\right) & \leq r\left((e-k)^{-1}\right) r(k) \\
& \leq \frac{r(k)}{1-r(k)}<1 .
\end{aligned}
$$

Hence, by the proof of Theorem 3.1, we can easily see that the sequence $\left\{x_{n}\right\}$ is Cauchy.

By the completeness of $X$, there is $x^{*} \in X$ such that $x_{n} \rightarrow x^{*}(n \rightarrow \infty)$. To verify $T x^{*}=x^{*}$, we have

$$
\begin{aligned}
d\left(T x^{*}, x^{*}\right) & \leq d\left(T x^{*}, T x_{n}\right)+d\left(T x_{n}, x^{*}\right) \\
& \preceq k\left(d\left(T x^{*}, x_{n}\right)+d\left(T x_{n}, x^{*}\right)\right)+d\left(x_{n+1}, x^{*}\right) \\
& \preceq k\left(d\left(T x^{*}, x^{*}\right)+d\left(x^{*}, x_{n}\right)+d\left(x_{n+1}, x^{*}\right)\right)+d\left(x_{n+1}, x^{*}\right) .
\end{aligned}
$$

That is,

$$
(e-k) d\left(T x^{*}, x^{*}\right) \preceq k d\left(x^{*}, x_{n}\right)+(e+k) d\left(x^{*}, x_{n+1}\right) .
$$

Hence, from the condition $r(k)<\frac{1}{2}<1$ we get

$$
d\left(T x^{*}, x^{*}\right) \preceq(e-k)^{-1}\left(k d\left(x^{*}, x_{n}\right)+(e+k) d\left(x^{*}, x_{n+1}\right)\right) .
$$

Therefore, it follows from Definition 3.1, Propositions 3.1, 3.2, 3.3, and 3.6 that we have $d\left(x^{*}, T x^{*}\right) \preceq y_{n}$ where $y_{n}$ is a $c$-sequence in cone $P$. Hence, for each $c \gg \theta$ we have $d\left(x^{*}, T x^{*}\right) \ll c$, so $d\left(x^{*}, T x^{*}\right)=\theta$ by Lemma 2.2. Thus $x^{*}$ is a fixed point of $T$.

Now we prove the uniqueness of the fixed point. In fact, if $y^{*}$ is another fixed point, then

$$
\begin{aligned}
d\left(x^{*}, y^{*}\right) & =d\left(T x^{*}, T y^{*}\right) \\
& \preceq k\left(d\left(T x^{*}, y^{*}\right)+d\left(T y^{*}, x^{*}\right)\right) \\
& =2 k d\left(x^{*}, y^{*}\right) .
\end{aligned}
$$

Thus

$$
d\left(x^{*}, y^{*}\right) \preceq(2 k)^{n} d\left(x^{*}, y^{*}\right)
$$

for any $n \geq 1$. 
By Lemma 2.3 and the fact that $\left\|(2 k)^{n} d\left(x^{*}, y^{*}\right)\right\| \rightarrow 0(n \rightarrow \infty)$ (noting that by the condition $r(k)<\frac{1}{2}$ and Remark 2.4, $\left.\left\|(2 k)^{n}\right\| \rightarrow 0(n \rightarrow \infty)\right)$, it follows that, for any $c \in \mathcal{A}$ with $\theta \ll c$, there exists $N \in \mathbb{N}$ such that, for any $n>N$, we have

$$
d\left(x^{*}, y^{*}\right) \preceq(2 k)^{n} d\left(x^{*}, y^{*}\right) \ll c,
$$

which implies by Lemma 2.2 that $d\left(x^{*}, y^{*}\right)=0$, so $x^{*}=y^{*}$, a contradiction. Hence, the fixed point is unique.

Theorem 3.3 Let $(X, d)$ be a complete cone metric space over a Banach algebra $\mathcal{A}$ and let $P$ be the underlying solid cone with $k \in P$ where $r(k)<\frac{1}{2}$. Suppose the mapping $T: X \rightarrow X$ satisfies the generalized Lipschitz condition

$$
d(T x, T y) \preceq k(d(T x, x)+d(T y, y)) \quad \text { for all } x, y \in X
$$

Then $T$ has a unique fixed point in $X$. And for any $x \in X$, iterative sequence $\left\{T^{n} x\right\}$ converges to the fixed point.

Proof Let $x \in X$ be arbitrarily given and set $x_{n}=T^{n} x, n \geq 1$. We have

$$
\begin{aligned}
d\left(x_{n+1}, x_{n}\right) & =d\left(T x_{n}, T x_{n-1}\right) \\
& \preceq k\left(d\left(T x_{n}, x_{n}\right)+d\left(T x_{n-1}, x_{n-1}\right)\right) \\
& =k\left(d\left(x_{n+1}, x_{n}\right)+d\left(x_{n}, x_{n-1}\right)\right) .
\end{aligned}
$$

That is,

$$
d\left(x_{n+1}, x_{n}\right) \preceq(e-k)^{-1} k d\left(x_{n}, x_{n-1}\right) .
$$

As is shown in the proof of Theorem 3.2, $\left\{x_{n}\right\}$ is a Cauchy sequence, and, by the completeness of $X$, the limit of $\left\{x_{n}\right\}$ exists and is denoted by $x^{*}$.

To see that $x^{*}$ is a fixed point of $T$, we have

$$
\begin{aligned}
d\left(T x^{*}, x^{*}\right) & \preceq d\left(T x^{*}, T x_{n}\right)+d\left(T x_{n}, x^{*}\right) \\
& \preceq k\left(d\left(T x^{*}, x^{*}\right)+d\left(T x_{n}, x_{n}\right)\right)+d\left(x_{n+1}, x^{*}\right) .
\end{aligned}
$$

So we get

$$
d\left(T x^{*}, x^{*}\right) \preceq(e-k)^{-1}\left(k d\left(x_{n+1}, x_{n}\right)+d\left(x_{n+1}, x^{*}\right)\right) .
$$

Therefore, it follows from Definition 3.1, Propositions 3.1, 3.2, 3.3, and 3.6 that we have $d\left(x^{*}, T x^{*}\right) \preceq y_{n}$ where $y_{n}$ is a $c$-sequence in cone $P$. Hence, for each $c \gg \theta$ we have $d\left(x^{*}, T x^{*}\right) \ll c$, so $d\left(x^{*}, T x^{*}\right)=\theta$ by Lemma 2.2. Thus $x^{*}$ is a fixed point of $T$.

Finally, we prove the uniqueness of the fixed point. The proof is as same as that in Theorem 2.3 in [15] since it actually does not require the assumption of normality of the underlying solid cone. 
Remark 3.2 The method and technique to prove the inequality

$$
r\left((e-k)^{-1} k\right)<1
$$

in Theorems 3.2 and 3.3 in this paper are more simple than and different from those in Theorems 2.2 and 2.3 in [15].

We conclude the paper with two examples.

Example 3.1 Let $\mathcal{A}=C_{\mathbb{R}}^{1}[0,1]$ be the same as that in Example 2.2. Then the set $P=\{x \in$ $\mathcal{A}: x \geq 0\}$ is a non-normal cone in $\mathcal{A}$.

Let $X=\{1,2,3\}$. Define $d: X \times X \rightarrow \mathcal{A}$ by $d(1,2)(t)=d(2,1)(t)=e^{t}, d(2,3)(t)=d(3,2)(t)=$ $2 e^{t}, d(3,1)(t)=d(1,3)(t)=3 e^{t}$, for all $t \in[0,1]$ and $d(x, x)(t)=\theta$ for each $x \in X$ and for all $t \in[0,1]$. We find that $(X, d)$ is a solid cone metric space over Banach algebra $\mathcal{A}$. Further, let $T: X \rightarrow X$ be a mapping defined with $T 1=T 2=2, T 3=1$ and let $k \in P$ defined with $k(t)=\frac{1}{3} t+\frac{1}{2}$. By careful calculations one sees that all the conditions of Theorem 3.1 are fulfilled. The point $x=2$ is the unique fixed point of the map $T$.

Example 3.2 [15] Let $\mathcal{A}=\mathbb{R}^{2}$. For each $\left(x_{1}, x_{2}\right) \in \mathcal{A},\left\|\left(x_{1}, x_{2}\right)\right\|=\left|x_{1}\right|+\left|x_{2}\right|$. The multiplication is defined by

$$
x y=\left(x_{1}, x_{2}\right)\left(y_{1}, y_{2}\right)=\left(x_{1} y_{1}, x_{1} y_{2}+x_{2} y_{1}\right) .
$$

Then $\mathcal{A}$ is a Banach algebra with unit $e=(1,0)$.

Let $P=\left\{\left(x_{1}, x_{2}\right) \in \mathbb{R}^{2} \mid x_{1}, x_{2} \geq 0\right\}$. Then $P$ is normal with normal constant $M=1$.

Let $X=\mathbb{R}^{2}$ and the metric $d$ be defined by

$$
d(x, y)=d\left(\left(x_{1}, x_{2}\right),\left(y_{1}, y_{2}\right)\right)=\left(\left|x_{1}-y_{1}\right|,\left|x_{2}-y_{2}\right|\right) \in P .
$$

Then $(X, d)$ is a complete cone metric space over a Banach algebra $\mathcal{A}$.

Now define mapping $T: X \rightarrow X$ by

$$
T\left(x_{1}, x_{2}\right)=\left(\log \left(2+\left|x_{1}\right|\right), \arctan \left(3+\left|x_{2}\right|\right)+\alpha x_{1}\right),
$$

where $\alpha$ can be any large positive real number. Then we have

$$
\begin{aligned}
d\left(T\left(x_{1}, x_{2}\right), T\left(y_{1}, y_{2}\right)\right) & \leq\left(\frac{1}{2}\left|x_{1}-y_{1}\right|, \frac{1}{10}\left|x_{2}-y_{2}\right|+\alpha\left|x_{1}-y_{1}\right|\right) \\
& \leq\left(\frac{1}{2}, \alpha\right) d\left(\left(x_{1}, x_{2}\right),\left(y_{1}, y_{2}\right)\right),
\end{aligned}
$$

where the spectral radius of $\left(\frac{1}{2}, \alpha\right)$ satisfies $r\left(\left(\frac{1}{2}, \alpha\right)\right)<1$. In addition, all the other conditions of Theorem 3.1 are fulfilled. By Theorem 3.1, $T$ has a unique fixed point in $X$.

Remark 3.3 Since in Example 3.1 the underlying solid cone $P$ in the Banach algebra $\mathcal{A}$ is not normal, we can conclude that any of the theorems in [15] cannot cope with Example 3.1, which shows that the main results without the assumption of normality in this paper are meaningful. 
Remark 3.4 In Example 3.2, we see that the main results in this paper are indeed more different than the standard results of cone metric spaces presented in the literature.

\section{Competing interests}

The authors declare that they have no competing interests.

\section{Authors' contributions}

Both authors contributed equally and significantly in writing this paper. Both authors read and approved the final manuscript.

\section{Author details}

'Department of Mathematics and Statistics, Hanshan Normal University, Chaozhou, 521041, China. ${ }^{2}$ Faculty of Mechanical Engineering, University of Belgrade, Kraljice Marije 16, Beograd, 11 120, Serbia.

\section{Acknowledgements}

The authors are grateful to the referees and the editors for valuable comments and suggestions, which have improved the original manuscript greatly. The research is partially supported by Doctoral Initial Foundation of Hanshan Normal University, China (No. QD20110920).

\section{Received: 27 December 2013 Accepted: 17 April 2014 Published: 06 May 2014}

\section{References}

1. Huang, L-G, Zhang, X: Cone metric spaces and fixed point theorems of contractive mappings. J. Math. Anal. Appl. $332,1468-1476(2007)$

2. Rezapour, S, Hamlbarani, R: Some notes on the paper 'Cone metric spaces and fixed point theorems of contractive mappings'. J. Math. Anal. Appl. 345, 719-724 (2008)

3. Jiang, S, Li, Z: Extensions of Banach contraction principle to partial cone metric spaces over a non-normal solid cone. Fixed Point Theory Appl. 2013, 250 (2013)

4. Abbas, M, Rajić, VĆ, Nazir, T, Radenović, S: Common fixed point of mappings satisfying rational inequalities in ordered complex valued generalized metric spaces. Afr. Math. (2013). doi:10.1007/s13370-013-0185-z

5. Al-Khaleel, M, Al-Sharifa, S, Khandaqji, M: Fixed points for contraction mappings in generalized cone metric spaces. Jordan J. Math. Stat. 5(4), 291-307 (2012)

6. Gajić, L, Rakočević, V: Quasi-contractions on a nonnormal cone metric space. Funct. Anal. Appl. 46(1), 75-79 (2012)

7. Ilić, D, Rakočević, V: Quasi-contraction on a cone metric space. Appl. Math. Lett. 22(5), 728-731 (2009)

8. Kadelburg, Z, Radenović, S, Rakočević, V: Remarks on 'Quasi-contraction on a cone metric space'. Appl. Math. Lett. 22(11), 1674-1679 (2009)

9. Radenović, S, Rhoades, BE: Fixed point theorem for two non-self mappings in cone metric spaces. Comput. Math. Appl. 57, 1701-1707 (2009)

10. Janković, S, Kadelburg, Z, Radenović, S: On the cone metric space: a survey. Nonlinear Anal. 74, 2591-2601 (2011)

11. Çakallı, H, Sönmez, A, Genç, C: On an equivalence of topological vector space valued cone metric spaces and metric spaces. Appl. Math. Lett. 25, 429-433 (2012)

12. Du, WS: A note on cone metric fixed point theory and its equivalence. Nonlinear Anal. 72(5), 2259-2261 (2010)

13. Kadelburg, Z, Radenović, S, Rakočević, V: A note on the equivalence of some metric and cone metric fixed point results. Appl. Math. Lett. 24, 370-374 (2011)

14. Feng, Y, Mao, W: The equivalence of cone metric spaces and metric spaces. Fixed Point Theory 11(2), $259-264$ (2010)

15. Liu, H, Xu, S: Cone metric spaces with Banach algebras and fixed point theorems of generalized Lipschitz mappings. Fixed Point Theory Appl. 2013, 320 (2013)

16. Rudin, W: Functional Analysis, 2nd edn. McGraw-Hill, New York (1991)

17. $\mathrm{Liu}, \mathrm{H}, \mathrm{Xu}, \mathrm{S}$ : Fixed point theorem of quasi-contractions on cone metric spaces with Banach algebras. Abstr. Appl. Anal. 2013, Article ID 187348 (2013)

18. Kadelburg, Z, Pavlović, M, Radenović, S: Common fixed point theorems for ordered contractions and quasi-contractions in ordered cone metric spaces. Comput. Math. Appl. 59, 3148-3159 (2010)

19. Kadelburg, Z, Radenović, S: A note on various types of cones and fixed point results in cone metric spaces. Asian J. Math. Appl. 2013, Article ID ama0104 (2013)

20. Dordević, M, Dorić, D, Kadelburg, Z, Radenović, S, Spasić, D: Fixed point results under c-distance in tvs-cone metric spaces. Fixed Point Theory Appl. 2011, 29 (2011). doi:10.1186/1687-1812-2011-29 